V. I. TIKHONOV, dr. of tech. sciences, professor "ONAT", Odessa, O. $\boldsymbol{V}$. TYKHONOVA, teacher "ONAT", Odessa

\title{
THE MATH MODELING COGNITIVE ISSUES OF GENERAL SYSTEM THEORY
}

The paper focuses researches on general system theory. Related publications surveyed. Cognitive aspects of system math modeling studied. The issues of 'state-space' and 'spacestructure' discussed for a model of open system. The role of observer and 'dark matter' formalism determined in system analysis. Figs.: 2. Refs.: 28 titles. dark matter.

Keywords: general system theory; math modeling; cognitive aspects; space-structure;

\section{Introduction. The problem statement.}

Modeling ability is an important philosophical category in our understanding both biological structures and artificial 'smart machines'. Diverse living matter entities (cells, organisms or their communities) apparently inherit and develop their own models of the world in the form of instincts, acquired reflexes or intellect. Through natural selection, these models as a form of individual or collective intelligence obtain clear motivation to survive in a volatile environment. Any model of a system is actually embodied either in fine proteins or in physical matter. The higher intelligence of a certain system substance, the more complicated fine matter organization. In developed living organisms, fine structures are bound with peripheral and central nervous system. The artificial intelligent systems are driven by microprocessors connected with sensors and actuators.

The great variety of biological individuals and intelligent technical devices determines a huge number of models. In fact, any science, language, or parts of it are models in our human world. The nature of models in biological objects and systems still remains a mystery in many ways. However, advances in artificial intelligence help understand some of the general principles in fine biological matter construction. In this regard, math modelling methods in general systems theory (GST) are of high interest as particular instrument of mental activity. The classical approach often treats a system as a set of co-related elements (e.g. "A whole composed of relationships among the members", [1]). Also other specific variations of this view are known.

In this work, we primarily treat a "system" as a cognitive model of a material object, i.e. somebodies subjective vision on a real object in terms of co-related parts of the whole which are distinguished by the subject. Besides,

(C) V.I. Tikhonov, O.V. Tykhonova, 2019 
our focus set on a system math model of related experiment.

Experimental physics, as well as fundamental researches in math logic, pose new challenges to specialists and philosophers. Our understanding of material world models requires new rethinking upon their rigor and legitimacy. In particular, we speak about fundamental categories of "space" and "time". Among physicists, relativity and uncertainty of object's models have already been established $[2,3]$. Now, the role of subject-observer in math model of an experiment is an actual issue. Near a century ago, K.F. Gödel proved incompleteness of any formal theory or math model [4], as well as confusion of the so-called 'naive sets theory' of G. Cantor $[5,6]$.

In parallel, J. Neumann formulated his multilevel sets theory ([7]) along with based on it math theory of quantum mechanics in terms of operators in generalized Hilbert space [8]. In recent decades, much attention of physicists is being paid to a relatively new phenomenon of "dark matter", which is hardly detected but seriously influences observed processes [9].

The practice shows, that academic courses on general system theory are not always in trend of cutting edge experimental or theoretical physics. The present work pretends to rethink the role of subject-observer and dark matter in system analysis and modeling. On this premise, some cognitive principles of systems' math modeling introduced, as well as a simple case of enhanced tensor approach for an abstract system.

\section{Related publications survey.}

In 1963, the book of the American mathematician G. Kron "Diakoptics" was published, which sets out original methods for the study of complex systems of different nature in parts [10]. Based on the decomposition of a complex system, topological models are built - graphs of the original system. Kron introduced the concept of space-structure. Such a space is described by a fully-connected graph of a network whose nodes are separate parts of the system (subnets). The matrix of such a graph contains in diagonal cells descriptions of individual subsystems. The off-diagonal elements describe the connections between the parts of the whole. For any subsystem, an analysis is carried out separately and a solution is sought. The overall solution is obtained by articulating the resulting partial solutions for all the subsystems.

The Kron's method is convenient for direct calculations on computers. This approach is also interesting because Kron first proposed a non-standard tensor analysis of electrical machines and networks, which can be used to study complex systems in other realms. Of course, the diakoptics does not provide general solution for various problems; therefore, each given case requires particular consideration.

The fundamental work on general systems theory is the book of L. Bertalanffy ([11], 1969). In the introduction, the author writes: "Systems 
theory ... is preeminently a mathematical field, offering partly novel and highly sophisticated techniques, closely linked with computer science, and essentially determined by the requirement to cope with a new sort of problem that has been appearing". The author focuses three basic math sections of GST: set theory, graph theory, network theory (p. 21). He further emphasizes the importance of such areas of mathematics as information theory, automata, game theory, decision making and queuing theories.

Finally, Bertalanffy identifies the least formalized part of the GST verbal models, i.e. models in terms of professional languages (pp. 22-23). He says: "A verbal model is better than no model at all or a model which, because it can be formulated mathematically, is forcibly imposed upon and falsifies reality. Theories of enormous influence such as psychoanalysis were unmathematical or, like the theory of selection, their impact far exceeded mathematical constructions which came only later and cover only partial aspects and a small fraction of empirical data. Mathematics essentially means the existence of an algorithm which is much more precise than that of ordinary language. History of science attests that expression in ordinary language often preceded mathematical formulation, i.e., invention of an algorithm".

Bertalanffy underscored the structural/functional hierarchy in our vision the world: "A similar hierarchy is found both in "structures" and in "functions". In the last resort, structure (i.e., order of parts) and function (order of processes) may be the very same thing: in the physical world matter dissolves into a play of energies, and in the biological world structures are the expression of a flow of processes. At present, the system of physical laws relates mainly to the realm between atoms and molecules (and their summation in macrophysics), which obviously is a slice of a much broader spectrum. Laws of organization and organizational forces are insufficiently known in the subatomic and the super-molecular realms. There are inroads into both the subatomic world (high energy physics) and the super-molecular (physics of high molecular compounds); but these are apparently at the beginnings. This is shown, on the one hand, by the present confusion of elementary particles, on the other, by the present lack of physical understanding of structures seen under the electron microscope and the lack of a "grammar" of the genetic code". Chapter 6 of [11] focuses the openness of a system model as a key GST-concept, along with the "bio-creatures-toautomata" correspondence in terms of system entropy. There are also discussed the issues of social and history system modeling, as well as system models in psychology and psychiatry.

One of the founders of the modern formalized theory of dynamical systems is the Hungarian mathematician R. Kalman ("Topics in mathematical system theory", 1969, [12], part 1 - "Theory of Automatic Control", and part 4 
- "Algebraic Theory of Linear Systems"). The second part of the book (P. Falb) considers the basics of the optimal control theory based on Pontryagin maximum principle. The third part (Arbib) studies the automata theory. The algebraic linear analysis is primarily focused on integral and discrete Laplace transformations, Fourier harmonic analysis, etc. The algebraic structure of linear systems allows establishing relationships between the models of pulsed and continuous systems, as well as the theory of automata.

The Kalman's approach to dynamic system modeling aims describing the flow of causal relationships from the past to the future. Based on the "modules theory", the linear stationary systems considered with the discrete time and a finite number of input/outputs (aka "black box"). One of the wellknown models of that type is the Kalman's filter to predict dynamic object behavior. In contrast to Kalman's continuous time in dynamic system study, the section "Automata theory" by Arbib uses system states transition in discrete time dots with no fixed time-metric. Such an approach is truly fruitful to logical system study. Though, the complexity of Kalman's formalism prevents easy understanding of dynamic systems theory by undergraduates.

A fundamental academia work on GST is "Theory of hierarchical multilevel systems" (M.D. Mesarović, D. Macko, Y. Takahara, 1970, [13]). This book targets production/economical applications (e.g. planning, operational management, accounting etc.). With respect to general aspects of system modeling, of great interest is multi-level hierarchy approach to complex systems study and system math modeling. In fact, the idea of world hierarchical order originate in deep antiquity (the ancient teachings of India, Egypt, Greece and Rome, etc.)

The works by J.Gigch from University of California ([14], 1978) laid a bridge between the general theory of systems as a new scientific discipline and system approach to practical tasks. Application sphere of these ideas embraces decision making in administrative-state apparatus and organizations. The goal setting, planning and optimization are key aspects in this work. The notion of a 'system' is introduced as a set of parts or components that are interconnected organizationally. The Gigch's works benefit study of system theory cognitive problems (e.g. optimization math task) on a qualitative and verbal level, avoiding cumbersome formalisms. This attracts a wide range of students and specialists, including the humanitarian sphere.

The chapter 8 of the first Gigch's book [14] ("Quantitative definition and measurement") introduces 'measurement through a hierarchy of nested models'. Chapter 9 ("Measurement Problems and Social Sciences") uses the methodology of fuzzy sets to formalize GST models.

A known propagandist of Kalman's dynamical systems theory is J.Casti (Univ. of New York). His dedication to "Connectivity, complexity, and 
catastrophe in large-scale systems" ([15], 1979) is: "To Rudolf Kalman, who transformed systems theory from a mystical art into a mathematical discipline". Casti noted: "One of the most pervasive and least well-defined concepts in modern intellectual thought is the notion of a system. One of the primary goals of this monograph is to provide a basis for common discussion of system-theoretic issues by presenting numerous examples of system problems from many disciplines and showing how they all may be described and analyzed by means of a rather small number of abstract paradigms".

The core Casti's idea is representation the system framework as a graph, which echoes the principles of Kron's diakoptics. In this case, a new concept of geometric space arises, the dimension of which is determined by the number of vertices of the graph. Also, the problem of the system complexity and its quantification is discussed. The concept of static and dynamic complexity of system behavior in time is introduced. Besides, the complexity of object management discussed in the context of its computational operability. An important role underscored to the qualitative (structural) and quantitative (algebraic) system framework.

The notable contribution to GST made E. Nappelbaum by Russian translations of Kalman, Feynman, Mesarovic and other known scientists in system analysis. Nappelbaum is also a co-author of "System Analysis-1979" journal $([16], 1980)$. In his preface to the second edition of the math theory of systems by R. Kalman [11], he wrote the following (for correct citation we also bring original text in Fig. 1). "From my point of view, the systems approach is based on cognition that any task is not something objective, existing regardless of who wants to solve it, but in some sense equally reflects as objective properties of reality, within which this task is attempted to be solved, and the attitude to this reality of the one who sets such a task...".

\footnotetext{
С моей точке зрения, системный подход зиждется на осознании того, что всякая задача не является чем-то объективным, существуюшим независимо от того, кто ее хочет решать, а в каком-то смысле в равной степени отражает как объективные свойства реальности, в рамках которой эту задачу пытаются решать, так и отношение к этой реальности того, кто такую задачу ставит. Иными словами, всякая реальность дает почву для постановки целого ряда различных, возможно несовместимых, а зачастую и прямо противоположных задач, и поэтому весьма важно не только корректно поставить задачу, но и отчетливо осознать, почему именно этой задаче мы отдаем предпочтение в нашем взаимодействии с реальностью. Системный анализ и другие методы этой же категории и должны помочь осознать, какие же из задач, которые в данной реальности можно поставить и решить, соответствуют желаниям и возможностям субъекта, намеревающегося изменить эту реальность, и позволить преобразовать несисгематизированные представления о реальности в постановку задачи, доступную для рационального решения формальными методами.
}

Fig.1. Original Russian text of the cited fragment 
"... In other words, any reality gives rise to the formulation of a number of different, possibly incompatible, and often directly opposing tasks, and therefore it is very important not only to set the task correctly, but also to clearly understand why it is this task that we prefer in our interaction with by reality. Systems analysis and other methods of this category should help to realize which of the tasks that in this reality can be set and solved correspond to the desires and possibilities of the subject intending to change this reality and allow unsystematic representations of the reality in the formulation of the problem, available for a rational solution by formal methods. And at the same time, the mathematical theory of systems, to which the book offered to you is devoted, is engaged in clarifying the properties of the formal description (models) of reality, which allow us to set problems and solve them. It is this close attention to the relationship between the subject of observation and its object that systems theory differs from ordinary mathematics, and this is where the center of its specific research lies".

The book "System analysis and design" (A. Dennis et al, [17], 2012) encourages GST-learning students not just to read about, but understand related issues, with a focus on the 'Unified modeling language' (UML-2) and "Software development lifecycle" (SDLC) as proper instruments for objectoriented analysis and design. Also, emphasized the visual-form interpretation throughout of the discussed issues to help students better understand the major elements and their relationships of the subject. Hereby, no specific math tools are discussed.

In [18] (2014) there are considered electrical, mechanical and hydraulic systems, along with their transfer functions estimation in MATLAB SIMULINK. The functional state-space aspects discussed with respect to differential equation in math models. The publication [19] (2014) discusses the state-space representation of a system in terms of tensors. It is reasoned, that tensor approach is directly applicable in concurrent linearly coupled dynamical systems for case study of distributed signal processing in cyber physical systems.

The work "Generic Framework for Simulation of Cognitive Systems" ([20], 2014) presents a model of a cognitive system based on communicating agent's population. There is underscored the importance of the environmental structure, in which a system evolves and learns, as well as the learning capacities of individual agents. The agent-based model shows that boundaries might be seen as a product of agent's communication in a given environment.

The work [21] (2016) presents the adaptation of Kron's tensor analysis of network for the electromagnetic compatibility design and analysis. The idea is, firstly decompose a complex system onto the sub-systems related to 
each other in graph view. Secondly, to map the system graph onto an abstract geometrical space, where the graph edge's weights play the role of independent (orthogonal) axes. The author claims that Kron's tensor model of electro-magnetic circuits based on space-structure geometrical approach were applicable to many other complex objects study.

The publication [22] (2017) exhibits the multilayer tensor factorization method with respect to recommender systems applications in E-commerce and entertainment industries. Tensors are understood as multi-dimensional arrays. The streaming tensor factorization method is considered in [23] (2018) for processing of high-volume and multi-way temporal data in Internet networks, recommender systems and image analysis. The paper presents the Bayesian robust streaming tensor factorization model to identify sparse outliers, automatically determining the underlying tensor rank and fitting the low-rank structure. The model is implemented in MATLAB. This approach is applied to complete streaming tensors in synthetic data, dynamic magnetic resonance imaging (MRI), video sequences and Internet traffic data.

S. Moura ([24], (2018) seeks to write non-trivial ordinary differential equations (ODE) for energy system. Once sustainable ODE properly defined, the system dynamics analyzed in terms of 'state-space' canonical template. Discussed transition from nonlinear object description to a linear systems of ODE.

The publication [25] (2018) calculates the many-body dynamics of polar-tonic states supported by an optical cavity, which is filled with organic molecules. Optical, vibration, and radioactive processes are treated on an equal footing, employing the algorithm of time-dependent matrix states. Also there analyzed single- and many-molecule cases to show the role of collective motion of molecular nuclei and dark states in determining the polar-ton dynamics and photon emission.

The work published by F. Schröder et al from Cambridge University [26] (2019) presents the tensor network simulation approach for multienvironmental open quantum dynamics via machine learning and entanglement renormalization. The authors stated that simulation of open quantum dynamics is a critical tool for understanding the non-classical properties of matter towards their functionalized presentation in future devices. However, this task is challenging due to strong non-Markovian coupling of environmental molecular vibrations to the electronic system degrees of freedom. For this purpose, an advanced computational strategy has been presented to allow tensor network methods computing the non perturbative, real-time dynamics of molecules. There exhibited an "ab-initio" machine learning and entanglement analysis to provide real-time insight of dissipative photo physics. 


\section{Discussion on publication survey. Objectives}

The survey of GST-researches indicates an obvious need in further developing of general systems theory as an academic discipline addressed to a wide range of applications from physics to humanitarian and social sciences. Now, diversity of methods in system analysis is exhibited. Engineering designers often construct highly abstract math models that weakly understandable even for specialists. Researchers on economics, sociology and other realms prefer less formalized methods of system analysis, more easily accepted by undergraduates.

Maybe the number one issue in system analysis is how to understand the "system" as a key formal term. Today, the multilevel hierarchy of a system vision increasingly prevails among the GST-specialists meaning that any stratum of a system can be uniformly presented on common principles but filled in with specific content. Also the concept of system model openness is more and more popular in contrast to the commonly known assumption of a "closed physical system". Of course, the axiom of a "closed system" is sometimes relevant to given task; however, with more comprehensive analysis, one sooner or later challenges the possibility of building the so-called closed system. An example of term confusion by "closed system" is known paradox about the "Universe thermal death' which allegedly follows from the thermodynamics' second law (aka 'entropy of any closed system invariably increases'). Yet, this paradox may be obviously resolved through the openness declaration of any system, which eliminates any logical statement about the "whole Universe" itself (except, perhaps, recognition the fact it actually exists but never completely seen). Remember the Bible commandment "Do not mention the name of your Lord in vain". Thus, the observable world around is only a finite open part of an infinite Universe, and for this visible part, the thermodynamics second law is not valid. It turns out that an open system can reduce its entropy by interacting with the rest of the world (which is what is actually happening). Apparently, this insight of an 'open system' is fruitful to other systems and models study (biological, economic, social etc.). Thus, "black box" as a popular dynamic system model is disputable under the doctrine of system model openness.

Another key aspect of GST-methodology is formalization the notion "state-space" of system, as well as the "spatial" and "time" categories mutual correspondence. In this respect, some questions of interest arise. First, are spatial and time coordinates crucially different or they can be joint to a uniformed 'space-time' framework? Second, what about the spatial and time coordinates dimensionality? Third, is time-argument as reversible as all the spatial coordinates are? Again, is a straight unidirectional arrow an adequate geometric model of time-axis? Our conventional world's spatial image is the 
notorious three-dimensional Cartesian space. In theoretical physics, also known more complicated forms of real matter existence, e.g. four-dimensional Minkowski space-time (with real Cartesian coordinates and imaginary time axis, [27], 1908). Minkowski space-time is a pseudo-Euclidean (or complex stratified) Euclidean space. Later on, new models of the physical world appeared (1921, T. Kaluza, 5-dimensional model; 1990s, E. Witten et al, 11dimensional model). In 1996 the famous book issued "The nature of space and time" (S. Hawking and R. Penrose [28]). Herein Hawking uses the term "Universe" meaning "all that is in nature" with no boundaries; next he concludes that Universe is closed. Also he made a cautious assumption of possibly looped time, but himself abandoned this idea a little bit later. With respect to coauthor's cognitive discussion around this book, the Penrose's theses about the quantum-wave model of gravity and tensor description of physical world, undoubtedly are significant steps on further generalization of previously known scalar and vector field models. Unlike his coauthor, Penrose prefers talking about the 'open universe', and later on uses the term "our Universe". Notably, that both coauthors do not argue the issues of physical space dimensionality beyond the four-dimensional Minkowski space-time.

Regarding the mathematical state-space of an arbitrary physical or humanitarian system the specialists primarily appeal to objective characteristics and do not underscore specific nature of modelling object in the context of somebody's subjective motivation. In this sense, position of Nappelbaum in [16] concerning the principal role of a subject-observer is of particular interest to our discussion. Besides, some recent researches in physics indicate increasing interest in the so-called 'Dark matter' phenomenon. A coherent idea was expressed in [26] regarding the "Dark states" in the tensor model of a technical system. However, the concepts of dark matter and dark states are less reflected in humanitarian system researches.

The present work objective: substantiation cognitive principles of math modeling physical objects, based on the open system architecture with subjectobserver and dark matter formalisms.

\section{Model as a subject's point of view on the object of study}

Known literature on general system theory (GST) commonly treats "system" as a set of elements of a whole related to each other. Such insight of system needs two remarks: a) system is a closed and single-level framework (no outside interfaces declared, and all the elements are peer-to-peer members); b) neither 'subject-observer' nor "dark-states" explicitly posed among the system elements. Consider above remarks in detail. 


\section{Openness and system hierarchy}

Is the system hierarchy inner property of a real object, or it is just our perception of it? The answer to this question seems not really actual but more important to simplify our mental image of a system complexity. The hierarchical model presentation of a great object allows the use of simple operational tools to handle subsystems at different levels. Recall the ancient teachings of Hermeticism philosophy (Tábula smarágdina): "What is below is like what is above. And what is above is similar to what is below. And this must be known in order to gain knowledge of the most wonderful One".

Apparently, the openness and multilevel system's hierarchy are rather mutually bound characteristics of our insight a large object, then its inherent property. Next, we distinguish the "openness in breadth" (i.e. up the hierarchy) and "openness in depth" (i.e. down the hierarchy). An arbitrary subsystem layer we assume as "zero-rank" stratum. In accordance with multilevel set theory by Neumann (i.e. von Neumann classes) introduce the following system model architecture. The up/down hierarchy subsystems layers (in comparison to predetermined zero-rank level) we'll define as positive/negative rank system strata. The "n-rank subsystem" integrates "n-rank elements". Each "n-rank element" may turn to "n-1-rank subsystem".

Therefore, each subsystem or element of rank $n$ is potentially open up and down in a system model hierarchy. In other words, any model of a system looks a "multi-slice cake" in the intermediate range between the observed microcosm and the macrocosm. Everything else but this "cake" remains beyond our perception and modeling, while new experiments extend the scope and horizons of our observations. Yet, anyone can solely observe a vanishingly small part of the whole infinite World. The multilevel hierarchical approach is applicable not solely to pure physical objects, but more in general for many other ones (informational, biological, social, economic etc.). The paradigm of open system hierarchy leads to the principal dualism in system architecture, i.e. each subsystem should have not one, but at least two types of presentations: a) subsystem opened "in depth" as a framework built on the set of inner elements and outer interface points; b) subsystem opened "in breadth" as a point of the overlying hierarchy level; this point potentially has a "hidden" inner structure.

\section{The role of subject-observer}

The inclusion observer into a system model inevitably makes it subjective, which can be perceived as its flaw. One can understand an inquisitive researcher who intuitively aims building an accurate object's model independent of a particular point of view. This is somehow possible in middle rank stratum observations between the microcosm and macrocosm with respect to our tools. Instead, elementary particles as objects of interest in 
quantum mechanics, inevitably change their behavior forced by the subject's observation instruments. Therefore, one cannot know the "real behavior" of tiny particles. In a quantum-physical experiment, we solely observe some results of our instrumental intervention into the physical matter. This means that a smart model of a system must necessarily include a "subject" as an actual participant in the process of experimental research and modeling. It also follows that any model of such a physical system is nothing more than subjectspecific formalization of a particular experiment (or a typical experiment, if it can be repeated under identical conditions). Each such experiment (and, hence, its "system model") pursues a well-defined goal, solves a limited circle of problems, beyond which it loses its relevance.

\section{Spatial-temporal system states}

In theoretical physics, a model of a four-dimensional pseudo-Euclidean space-time of three real Cartesian axes and one imaginary time axis (with Minkowski metric) is often considered. Also there are known models with greater dimensions (e.g. 10-dimensional space in string theory).

In engineering applications are used the so-called abstract multidimensional spaces (e.g. N-dimensional Euclidean space). Functional analysis widely explores infinite-dimensional spaces (e.g. Hilbert space). In our opinion, everything that concerns the questions of infinity requires more discussion towards logical correctness and expediency under the modern quantum insight of matter.

How many measurements (coordinate axes) are necessary and/or sufficient to describe both static and dynamic states of an arbitrary system? After all, it is constructively impossible to build models with an infinite number of dimensions. Maybe we should abandon the "infinite-dimensional spaces" in general in the theory of mathematical modeling? What is the essence of the concept of "time"? Is time objectively existing entity? Is time continuous, unidirectional and one-dimensional matter? A reasoned response to each of these questions may require considerable efforts. Without claiming the ultimate completeness, we will formulate some postulates and principles to introduce a system math model in terms of tensor study of complex systems in parts.

\section{Time category in system models}

Apparently, any observation or measurement requires finite time. Therefore, the concept of the "instantaneous" state of the system at the "point" of time $t$ we will try to avoid in our math models of systems (considering it only as a theoretical idealization). The time category we will treat mostly as topological coordinates (i.e. discrete consecutive observation intervals, not necessarily of the same magnitude). So, instead of real time, we will simply describe system transitions from one state to another. Time itself next 
understood as tags of consequent system states (such a time model is used, for example, in Markov chains). Unlike known models with one-dimensional time, we consider time hierarchy nested intervals of various scales. A system model as an abstract math image of a physical experiment we will consider time as a closed chain of events in which the end time point coincides with initial point by all the controlled factors. This means that, up to unrecorded parameters, the time in a system model will be considered cyclically closed and reversible, i.e. peer the other "purely spatial" coordinates. As an instant static state of system $S$, we will treat system's averaged state on a discrete observation interval of minimal scale.

\section{State-space of system model}

Suppose system $S$ an object observed by $N$ sensors each having own freedom. If all the sensors' readings are pair-wise independent, then the set of object's states maps onto a certain volume of an abstract $N$-dimensional space $V$ with orthogonal axes ( $N$-dimensional parallelepiped in $N$-dimensional Euclidean space).

Such a model is often used in applied researches. Though, once sensors readings bound, the non-orthogonal Euclidean space $U$ shall be applied, which defined by the so-called Riemann metric tensor $R$ (square positive Hermitian matrix of dimension $N \times N)$. The geometrical meaning of Riemann tensor $R$ is the scalar product of basis vectors (as a formal description of the relationship between all $N$ sensors). If sensor properties and relationships change in time, the so-called local Euclidean space can be used as a tensor function of a "point" (where a "point" understood as different temporal/spatial position of the object).

Is there any fundamental restriction on the number of independent (or at least partially independent) sensors when observing a complex object? Most likely not, therefore we assume that a system $S$ may have a state space of any finite dimension $N$, depending on the nature of experiment. The states of the system will also be considered discrete points (each of which symbolizes a local uncertainty domain in the state-space), since nothing can be precisely measured. In addition, the fact that the system is in a particular state will also not be considered obvious, but only expected with some probability. The dynamic properties of a system with a similar model are described by unconditional probabilities of states, as well as by conditional probabilities of transition from one state to another. The simplest model of this kind is the scalar function of probability distribution for various object states. This model is valid if none previous states impact future ones.

The model of such a system is one-dimensional array (aka "vectorstring") of $M$ probabilities ( $M$ is the number of discrete states in the $\mathrm{N}$-dimensional state-space). In a more complicated (but nonetheless, fairly 
simple) case, the probability of system state transition depends only on its current state (the so-called Markov discrete random process or a Markov chain). The Markov chain can be described by a square transition matrix $H$ of size $M \times M$. The diagonal cells of matrix $H$ contain unconditional states' probabilities; the off-diagonal elements determine conditional probabilities of state transitions. If $H$ is a positive symmetric matrix, it satisfies the Riemann metric tensor. If the object's dynamic properties gradually evolve over a larger time scale, the model of system $S$ is a looped sequent time-chain (i.e. discrete topological circle) of space-tensors. This space-tensor may be of different rank and complexity. The simplest case is zero-rank tensor presentation (scalar function of discrete time). The deeper probability dependence of system state transitions, the more complex dynamic model of the system looks. More advanced is the first-rank tensor view (vector-function of discrete time). If Markov dynamic system considered, then second-rank tensor applicable (i.e. matrix of states transitions as a function of discrete time).

In the second approximation, the conditional transition to each state depends on two previous states. So, the third-rank tensor shall be used for such a non-Markov process modeling (i.e. $M \times M \times M$-dimensional table of conditional probabilities for state transitions as a function of discrete time).

Consider the mentioned above multidimensional looped chain in a dynamic system model. The looped time-chain is discrete topological circle which ideal image is continuous geometrical circle. When multilevel-time is used (of nested time-circles of different scales), then geometrical image of such time presentation evolves to multidimensional torus. The fact is known from general topology, that torus is a unique closed manifold, where ubiquitously continuous vector (or tensor) field can be defined. This property of torus is important for tensor modeling of dynamic objects with multidimensional looped time.

Above approach with some variations, remains popular and acceptable for system modeling in certain tasks. Though, it neither provides the system hierarchical architecture nor subject-observer's important role in physical system modeling. An abstract $N$-dimensional space of object-states model is formed by subject's instruments of observation, as if they did not impact the object observed. These instruments and related state-space look as the "Absolute" towards an object of study (e.g. space is empty if no objects detected). Such vision is challenging in microcosm study, while sensors roughly impact tiny things. For a broader scope of general system theory, the model of passive observation of objects needs enhancement.

\section{Space-structure as a generalized system model}

Considered above state-space of a physical system (denote it $\Phi$ ) assumes to be a priory and stable, wherein a system instant state looks like an abstract 
"dot", and system behavior in time as a phase-loop of trajectory in $\Phi$. In Kron's tensor analysis, as well as some other works followed him, a fundamentally new category (aka "space-structure") was developed in the theory of math models. Some distinctive features of the "space-structure" in contrast to common "phase state-space" of a physical system are discussed further on. In fact, the "space-structure" concept formalizes the current perception the 'objects of interest' by a "subject-observer". Herewith, the following cognitive categories emerge: $S$ - "subject-observer" which understood as a class of possibly multiple observers; $X$ - class "objects of interest" with respect to given observer; $W$ - class of "dark matter" entities around observer. Relationships among $S, X, W$ in time spot $t$ denote $\mathfrak{R}_{t}$ and present as a graph in Fig. 2. This graph reflects a single stratum of some rank in a multilayer hierarchy of a system model. Each of the three classes $S, X, W$ may include one or more elements, related to each other.

Compare to conventional system models with "state-space" formalism built on the objects of class $X$, the graph in Fig.2 includes two additional categories (i.e. class $S$ of subject-observers and class $W$ of dark matter entities). While "state-space" records typically emerge by sensor readings, the only quantitative data supposed in "space-structure" observations are on-pair relations estimation among the members of specified three classes $(S, X$ and $W)$.

On this premise, the "space-structure" appears as mapping relationships $\mathfrak{R}_{t}$ onto an abstract geometrical form. An instant geometric image of "spacestructure" might be a set of vectors (rapper), where each vector corresponds to an object from classes $S, X$ and $W$.

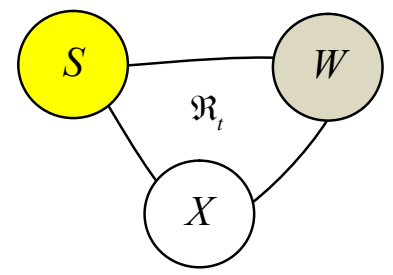

Fig. 2. Relationships among subject, object and dark-matter

Any given set of real vectors $V$ can be determined in diverse forms, e.g. an algebraic array of vector modules along with the matrix of correspondent angles among them; matrix $H$ of scalar product $[V \times V]$; matrix $V$ of normal coordinates in an orthonormal Euclidian basis; matrix of normal coordinates in non-orthogonal Euclidian basis; matrix of either covariant or contra-variant tensor components in non-orthogonal Euclidian basis with known metric). 
All these forms of vector-rapper presentations are equivalent, and therefore, can be obtained from each other with some reservations. So, a geometric rapper view of a "space-structure" can be taken from the weighted graph of relationships among the classes $S, X$ and $W$. Detail consideration of these issues will be the subject of our next publications.

\section{Conclusion}

Despite significant advances in system analysis realm, the general systems theory is far from complete as an academic discipline. The difficulties in general theory of systems design are due, among other things, to a wide range of applied system researches - from quantum physics to economics and the humanities. In present work, another attempt is made to further formalize the general theory of systems based on key principles of modern physics and mathematical logic, such as openness and multilevel hierarchy of system architecture, the uncertainty of object's state observations and measurements, relativity concept. The paper also focuses the cognitive aspects of such metaphysical categories as 'temporal and spatial structure' with respect to real world object modeling. In particular is underscored the so called 'spacestructure' framework in tensor presentation of experimental data, as well as the principal role of subject-observer in an open system model. In addition to subject-observer, the dark matter formalism has been introduced as extra member of system tensor model.

\section{References:}

1. Definitions \& Translations [Electronic resource]. - Available: https:// www.definitions.net/ definition/system.

2. General relativity [Electronic resource]. - Available: https://science.jrank.org/pages /5790/Relativity-General.html.

3. The Uncertainty principle [Electronic resource]. - Available: http://230nsc1.phyastr.gsu.edu/hbase/uncer.html.

4. Gödel K. (1913), Über die Vollständigkeit des Logikkalküls, Doctoral dissertation, Univ. of Vienna. - 1929. / A. Einstein, M. Grossmann // Zeitschrift für Mathematik und Physik, vol. 62, pp. 225-261.

5. Cantor, G. (1883), "Ueber unendliche, lineare Punktmannichfaltigkeiten", Mathematische Annalen, 21 (4), pp. 545-591.

6. Russell, B. (1980), The Principles of mathematics, $798 \mathrm{p}$.

7. Neumann, Von J. (1927), "Mathematische Begründung der Quantenmechanik", Nachrichten von der Gesellschaft der Wissenschaften zu Göttingen, MathematischPhysikalische Klasse, pp. 1-57.

8. Neumann, Von J. (1928), "Die Axiomatisierung der Mengenlehre", Mathematische Zeitschrif, No. 27, pp. 669-752.

9. Antonov, A.A. (2016), Explaining the phenomenon of dark matter and dark energy by existence of the hidden multiverse [Electronic resource]. - Available: http://pubs.sciepub.com /faac/2/1/1/index.html.

10. Kron, G. (1963), Diakoptics. The piecewise solution of large scale systems, London, MacDonald, $166 \mathrm{p}$. 


\section{Вісник Національного технічного університету "ХПІ", 2019, № 13 (1338)}

ISSN 2079-0031 (Print) ISSN 2411-0558 (Online)

11. Bertalanffy, L. (1969), General system theory, George Braziller, New York, 289 p.

12. Kalman, R.E., Falb, P.L., Arbib, M.A. (1969), Topics in mathematical system theory, [Electronic resource]. Available: https://trove.nla.gov.au/work /21064526? selectedversion=NBD4945.

13. Mesarović, M.D., Macko, D., Takahara, Y. (1970), Theory of hierarchical multilevel systems, Academic Press. 294 p. - Available: https://epdf.tips/theory-of-hierarchicalmultilevel-systems.html.

14. Gigch, J.P. (1978), Applied general systems theory, HarperCollins College Div., 2 edition. $602 \mathrm{p}$.

15. Casti, J. (1979), Connectivity, complexity, and catastrophe in large-scale systems, JOHN WILEY \& SONS, Chichester - New York - Brisbane - Toronto, 203 p.

16. Nappelbaum, E.L. (1979), System analysis as a research program, System studies, Ejegodnik, Moskow, Science, 384 p.

17. Dennis, A., Wixom, B.H., Roth, R.M. (2012), System analysis and design, 5 edition. $594 \mathrm{p}$.

18. Patil, A., More, S. (2014), Mathematical modeling of physical system, [Electronic resource]. - Available: https://www.researchgate.net/publication /272713431 Mathematical_modeling_of_physical_system.

19. Murthy, G.R. (2014), Concurrent cyber physical systems: Tensor state space representation, [Electronic resource]. - Available: https:/arxiv.org/ftp/arxiv/ papers/1303/1303.1597.pdf.

20. Generic framework for simulation of cognitive systems: A case study of color category boundaries, 2014. [Electronic resource]. - Available: https://link.springer.com/chapter /10.1007/978-3-319-02309-0_42.

21. Maurice, O. (2016), Adaptation of Kron's tensorial analysis of network for the EMC design and analysis of systems, [Electronic resource]. - Available: https://hal. archivesouvertes.fr/hal-01382221/document.

22. Multilayer tensor factorization with applications to recommender systems, 2017. [Electronic resource]. - Available: https://arxiv. org/pdf/1711.01598.pdf.

23. Robust factorization and completion of streaming tensor data via variational Bayesian inference, 2018. [Electronic resource]. - Available: https://arxiv.org/pdf/1809.01265.pdf.

24. Moura, S. (2018), Energy systems and Control. Chapter 1: Modeling and systems analysis, [Electronic resource]. - Available: https://ecal.berkeley. edu/files/ce295/CH01ModelingSystems.pdf.

25. Tensor network simulation of non-Markovian dynamics in organic polaritons, 2018, [Electronic resource]. - Available: https://www. ncbi.nlm.nih.gov/pubmed/30547635/.

26. Tensor network simulation of multi-environmental open quantum dynamics via machine learning and entanglement renormalization, 2019, [Electronic resource]. - Available: https://www.ncbi.nlm.nih.gov/pmc/articles/PMC6401190/.

27. Minkowski H. (1908), The fundamental equations for electromagnetic processes in moving bodies, [Electronic resource]. - Available: https://en.wikisource.org/ wiki/Translation. 28. Hawking S.W. Penrose, R. (1996), The Nature of Space and Time, [Electronic resource]. Available: http://www.theory.caltech.edu/ preskill/pubs/ preskill-1996-hawking.pdf.

The article is presented by doctor of technical sciences, professor, the head of "Radio and Television" depart. of "O.S. Popov ONAT", Honored Worker of Science and Technology of Ukraine Gofiyzen O.V. 
Вісник Національного технічного університету "ХПІ", 2019, № 13 (1338) ISSN 2079-0031 (Print) ISSN 2411-0558 (Online)

Tikhonov Victor

Doctor of technical science, associate professor

Laureate of the Ukraine State Prize for Science and Technology,

Professor of Communication Networks department

O.S. Popov Odessa National Academy of Telecommunications

St. Kuznechna 1, Odessa 65029, Ukraine

Tel.: 067-752-13-90,E-mail: victor.tykhonov@onat.edu.ua

Tykhonova Olena

Teacher of Communication Networks department

O.S. Popov Odessa National Academy of Telecommunications

St. Kuznechna 1, Odessa 65029, Ukraine

Tel.: 097-490-56-18, E-mail: elena.tykhonova @onat.edu.ua 
УДК 303.732

Когнітивні проблеми математичного моделювання у загальній теорії систем / Тіхонов В.I., Тихонова О.В. // Вісник НТУ "ХПІ". Серія: Інформатика та моделювання. - Харків: НТУ "ХПІ". - 2019. - № 13 (1338). - С. 137 - 154.

Робота присвячена дослідженням у загальній теорії систем. Наведено огляд тематичних публікацій. Досліджено когнітивні аспекти математичного моделювання систем. Розглянуто питання "простору-станів" та "просторуструктури" у тензорній моделі відкритої системи. Визначено роль спостерігача $\mathrm{i}$ механізм формалізації "темної матерії" у системному аналізі. Іл.: 2. Бібліогр.: 28 назв.

Ключові слова: загальна теорія систем; математичне моделювання; когнітивні аспекти; простір-структура; темна матерія.

\section{УДК 303.732}

Когнитивные проблемы математического моделирования в общей теории систем / Тихонов В.И., Тихонова Е.В. // Вестник НТУ "ХПИ". Серия: Информатика и моделирование. - Харьков: НТУ "ХПИ". - 2019. - № 13 (1338). - С. 137 -154 .

Работа посвящена исследованиям по общей теории систем. Приведен обзор тематических публикаций. Исследованы когнитивные аспекты математического моделирования систем. Рассмотрены вопросы "пространствасостояний" и "пространства-структуры" в тензорной модели открытой системы. Определена роль наблюдателя и механизм формализации "темной материи" в системном анализе. Ил.: 2. Библиогр.: 28 назв.

Ключевые слова: общая теория систем; математическое моделирование; когнитивные аспекты пространства-структуры; темная материя.

UDC 303.732

The math modeling cognitive issues of general system theory / Tikhonov V.I., Tykhonova O.V. // Herald of the National Technical University "KhPI". Series of "Informatics and Modeling". - Kharkov: NTU "KhPI". - 2019. - №.13 (1338). P. $137-154$.

The paper focuses researches on general system theory. Related publications surveyed. Cognitive aspects of system math modeling studied. The issues of "statespace" and "space-structure" discussed for model of open system. The role of observer and "dark matter" formalism determined in system analysis. Figs.: 2. Refs.: 28 titles.

Keywords: general system theory; math modeling; cognitive aspects; spacestructure; dark matter. 